\title{
Usefulness of serum miR-1246/miR-106b ratio in patients with esophageal squamous cell carcinoma
}

\author{
ISAMU HOSHINO ${ }^{1}$, FUMITAKA ISHIGE ${ }^{2}$, YOSUKE IWATATE ${ }^{2}$, HISASHI GUNJI $^{1}$, FUMIAKI SHIRATORI ${ }^{1,3}$, \\ NAOKI KUWAYAMA $^{1}$, YOSHIHIRO NABEYA ${ }^{1}$, NOBUYOSHI TAKESHITA ${ }^{4}$ and HISAHIRO MATSUBARA ${ }^{5}$ \\ ${ }^{1}$ Division of Gastroenterological Surgery and ${ }^{2}$ Department of Hepatobiliary and Pancreatic Surgery, Chiba Cancer Center, \\ Chuo-ku, Chiba 260-8717; ${ }^{3}$ Department of Surgery, School of Medicine, Toho University, Ota-ku, Tokyo 143-8541; \\ ${ }^{4}$ Division of Surgical Technology, National Cancer Center Hospital East, Kashiwa, Chiba 277-8577; \\ ${ }^{5}$ Department of Frontier Surgery, Graduate School of Medicine, Chiba University, Chuo-ku, Chiba 260-8670, Japan
}

Received June 4, 2020; Accepted September 8, 2020

DOI: $10.3892 /$ ol.2020.12213

\begin{abstract}
The function of microRNAs (miRs) is associated with the development and progression of various malignancies, with miRs presenting stably in the serum. The current study assessed the role of miR-1246 and miR-106b in the serum of patients with esophageal squamous cell carcinoma (ESCC). A comprehensive microarray analysis of miR expression was performed using the serum of patients with ESCC, which were subsequently validated via reverse transcription-quantitative PCR. A total of 55 test samples were obtained from Chiba University and 101 validation samples were gained from Chiba Cancer Center. The results revealed that miR-1246 expression significantly increased and miR-106b expression significantly decreased in each cohort. Receiver operating characteristic analysis revealed that the area under the curve (AUC) value of miR-1246 was 0.816 (sensitivity, $72.7 \%$; specificity, $69.2 \%$ ) and 0.779 (sensitivity, $71.3 \%$; specificity, $70.6 \%$ ) for the test and validation cohorts, respectively. The AUC of miR-106b was 0.716 (sensitivity, $65.5 \%$; specificity, $61.6 \%$ ) and 0.815 (sensitivity, 74.3\%; specificity, 73.5\%), respectively. In addition, the AUC of the miR-1246/miR-106b ratio was 0.901 (sensitivity, 80.0\%; specificity, 80.0\%) and 0.903 (sensitivity, $82.1 \%$; specificity, $82.3 \%$ ), respectively, which indicated a higher diagnostic ability compared with that of miR-1246 or miR-106b alone. The high miR-1246/miR-106b ratio group
\end{abstract}

Correspondence to: Dr Isamu Hoshino, Division of Gastroenterological Surgery, Chiba Cancer Center, 666-2 Nitona-cho, Chuo-ku, Chiba 260-8717, Japan

E-mail: ihoshino@chiba-cc.jp

Abbreviations: ESCC, esophageal squamous cell carcinoma; miR, microRNA; ROC, receiver operating characteristic; AUC, area under the curve; ICC, intraclass correlation coefficient; CRC, colorectal cancer

Key words: microRNA-1246, microRNA-106b, esophageal squamous cell carcinoma, serum was associated with clinicopathological factors such as depth of invasion, progression, lymph node metastasis, and poor prognosis. Therefore, effective biomarkers may be generated by combining individual miRs obtained by comprehensive analysis of ESCC patient sera.

\section{Introduction}

Esophageal cancer is one of the intractable gastrointestinal cancers. Its incidence and mortality are close, with recent statistics reporting 509,000 deaths out of 572,000 cases (1). Esophageal squamous cell carcinoma (ESCC) is the major pathological histology throughout Asia, whereas adenocarcinoma is major in Europe and the United States (2). Owing to advances in medical technology, cases of esophageal cancer when diagnosed early can be resolved using endoscopy. Therefore, the survival rate of patients with an early diagnosis of esophageal cancer and their quality of life, with esophageal preservation, has been greatly improved. In contrast, the overall survival rate of ESCC has been reported to be at most 20-30\%, with room for improvement (3). When detected early, the 5-year survival rate of patients with ESCC is $80-90 \%(4,5)$; however, early-stage esophageal cancer is less likely to show clinical symptoms, and a lack of reliable noninvasive screening methods has hindered its detection. Nevertheless, when ESCC is diagnosed relatively early, the prognosis is clearly good, and the method used for early diagnosis before the onset of symptoms is important. Therefore, to improve the prognosis of ESCC, an intractable cancer, it is necessary to search for a biomarker that can be detected even earlier $(6,7)$.

MicroRNA (miR), a non-coding RNA, is a functional RNA that is eventually edited to $19-22$ bases. This small RNA was discovered in Caenorhabditis elegans in 1993 (8), and its name was proposed in 2001 (9). Many miRs are involved in the onset and progression of various chronic diseases and malignant tumors. In particular, miRs associated with malignant tumors are classified into oncogenic miR (oncomiR) (10), a positively regulated $\mathrm{miR}$; tumor suppressor $\mathrm{miR}$, a negatively regulated miR. In addition, miRs are relatively stable in body fluids such as the blood by forming complexes with proteins and being included in microparticles such as exosomes. The usefulness 
of these miRs as biomarkers has been previously reported, and we have also reported the usefulness of miR-1246 as a biomarker in ESCC (11). miR-1246 is a biomarker not only in esophageal cancer but also in gastrointestinal cancers such as stomach and pancreatic cancers (12). Furthermore, in our previous report, miR-106b expression was significantly lower in patient sera than in that of healthy volunteers. miR-106b clusters together with miR-93 and miR-25, and has a tumor suppressor function in colorectal and ovarian cancers (13-15). In addition, its expression is reduced in the serum of gastric cancer patients (16-21).

The findings of our previous studies and other previous reports on other organ cancers suggest the usefulness of combination of miR-1246 and miR-106b as biomarkers. Here, we investigated the expression levels of miR-1246 and miR-106b in the serum of patients with ESCC and their clinicopathological significance.

\section{Materials and methods}

Ethical approval. Written informed consent was obtained from all patients, and the study was approved by the Ethics Committee of Graduate School of Medicine, Chiba University (no. 889), and Chiba Cancer Center (no. H29-0005) and performed in compliance with the Declaration of Helsinki.

Sample collection. Between April 2013 and April 2016, venous blood samples were collected from 55 patients with ESCC and 39 healthy individuals at the Graduate School of Medicine, Chiba University in Chiba, Japan. Between April 2016 and April 2019, venous blood samples were collected from 101 patients with ESCC and 34 healthy individuals at the Chiba Cancer Center in Chiba, Japan. These samples were collected before any therapies, including endoscopic resection, surgery, chemotherapy, or radiotherapy. Venous blood samples were centrifuged at $1,500 \mathrm{x} g$ for $5 \mathrm{~min}$ at $4^{\circ} \mathrm{C}$ to obtain serum. The serum samples were then stored at $-80^{\circ} \mathrm{C}$ until further processing.

RNA extraction. Total RNA was extracted from $200 \mu \mathrm{l}$ of serum using a miRNeasy Serum/Plasma Kit (Qiagen) according to the manufacturer's instructions. This kit contained the Caenorhabditis elegans cel-miR-39, which was used as a spike-in control.

Reverse transcription. Total RNA was reverse transcribed to cDNA using a miScript II RT Kit (Qiagen). In each reaction, $50 \mathrm{ng}(12 \mu \mathrm{l})$ of template RNA was combined with a master mix containing $4 \mu \mathrm{l}$ 5x miScript HiSpec Buffer, $2 \mu \mathrm{l}$ 10x miScript Nucleics Mix, and $2 \mu 1$ miScript Reverse Transcriptase Mix and incubated for $60 \mathrm{~min}$ at $37^{\circ} \mathrm{C}$. The reactions were then incubated for $5 \mathrm{~min}$ at $95^{\circ} \mathrm{C}$ to inactivate the miScript Reverse Transcriptase Mix and placed on ice.

miRNA microarray. Total RNA (100 ng) was labeled and hybridized following the Human microRNA Microarray Kit protocol and using the Human miRNA Microarray Kit (Release 16.0; Agilent Technologies). Hybridization signals were detected with a DNA microarray scanner G2505C (Agilent Technologies), and the scanned images were analyzed using the Agilent Function Extraction Software program (v10.7.3.1).
Normalization was performed using the Agilent GeneSpring GX software program version 11.0.2 (per chip: Normalization of control genes; per gene: None). The Agilent Human miRNA Microarray (Design ID: 031181) contained 1205 human miRs in total with 144 human viral miRs without control probes. The miR expression profile of the serum samples from patients with ESCC was examined using a microarray analysis of three patients and four healthy individuals. miR-16 was used as an endogenous control.

Reverse transcription-quantiative PCR (RT-qPCR). Quantitative RT-qPCR was performed using the miScript SYBR $^{\circledR}$-Green PCR Kit (Qiagen) in a 7300 Real-Time PCR system (Applied Biosystems; Thermo Fisher Scientific, Inc.). The expression levels of miR-1246 (assay ID: 462575) and miR-106b (assay ID: 000442) were analyzed using TaqMan quantitative real-time PCR (TaqMan MicroRNA assays; Applied Biosystems; Thermo Fisher Scientific, Inc.) and normalized to the levels of cel-miR-39 (assay ID: 001093). The parameters of the PCR reaction were as follows: $95^{\circ} \mathrm{C}$ for $15 \mathrm{~min}$ followed by 40 cycles of $94^{\circ} \mathrm{C}$ for $15 \mathrm{sec}, 55^{\circ} \mathrm{C}$ for $30 \mathrm{sec}$, and $70^{\circ} \mathrm{C}$ for $34 \mathrm{sec}$. All reactions were performed in duplicate. Relative expression was calculated using comparative cycle threshold $(\mathrm{Ct})$ values. Relative miR-1246 or miR-106b expression was calculated using the $2^{-\Delta \mathrm{Ct}}$ method, where $\Delta \mathrm{Ct}=\mathrm{Ct}$ (miR-1246 or miR-106b)-Ct (cel-miR-39) (22).

Statistical analysis. Data was conformed to a normal distribution using Shapiro-Wilk's test. An unpaired Student's t-test was performed to compare differences in age. A Mann-Whitney U test was performed to compare differences in miR-1246 expression levels between patients with cancer and healthy individuals. Spearman's rank correlation coefficient was used to assess correlations between the miR-1246 expression levels in the three body fluids. The $\chi^{2}$ test or Fisher's exact probability test was used to evaluate correlations between serum and miR expression levels and clinicopathological tumor factors. Receiver operating characteristic (ROC) curves and area under the curve (AUC) were used to assess the sensitivity and specificity of serum miR levels in detecting ESCC. All tests were two-sided, and the significance level was set at a P-value $<0.05$. The survival period of the patients was defined as the duration from the time of surgery to either death or the last follow-up day, and the survival rate was calculated using the Kaplan-Meier method. Comparisons of two groups as univariate analyses were performed using the log-rank test. JMP 14 (SAS Institute, Inc.) software was used for all statistical analyses.

\section{Results}

MiRs with large fluctuations in expression levels. A comprehensive expression analysis using microarray identified six miRs that were significantly upregulated and five that were significantly downregulated in ESCC serum samples compared with controls $(\mathrm{P}<0.05$; Table I). miR-1246 expression levels were most significantly upregulated in the serum of patients with ESCC, and miR-106b was the second most downregulated miR. The fourth most downregulated miR-93 forms a cluster with miR-106b, and thus miR-106b was considered 
Table I. Differentially expressed miRNAs in patients with esophageal squamous cell carcinoma.

A, Upregulated miRNAs

\begin{tabular}{lc}
\hline miRNA & P-value \\
\hline hsa-miR-1246 & 0.008 \\
hsa-miR-3202 & 0.032 \\
hsa-miR-23a & 0.038 \\
hsa-miR-718 & 0.042 \\
hsa-miR-3610 & 0.043 \\
hsa-miR-4271 & 0.044 \\
\hline
\end{tabular}

B, Downregulated miRNAs

\begin{tabular}{lc}
\hline miRNA & P-value \\
\hline hsa-miR-144 & 0.011 \\
hsa-miR-106b-5p & 0.034 \\
hsa-miR-486-5p & 0.039 \\
hsa-miR-93 & 0.049 \\
hsa-miR-451 & 0.050 \\
\hline
\end{tabular}

important. Therefore, miR-1246 and miR-106b were selected as candidates for further analysis.

miR-1246 and miR-106b expression in patients with ESCC. In total, 156 participants were recruited, 55 in the test cohort and 101 in the validation cohort. In both the test and validation cohorts, miR-1246 expression was significantly higher in patients with ESCC than that in healthy controls (Fig. 1A, B, D and E). In contrast, the expression of miR-106b was significantly lower in patients with ESCC than that in healthy controls (Fig. 2A, B, D and E).

Diagnostic ability of serum levels of miR-1246 and miR-106b. ROC curve analysis revealed the sensitivity of miR-1246 as a diagnostic indicator of ESCC (Fig. 1C and F). The AUC was 0.816 (sensitivity $72.7 \%$, specificity $69.2 \%$ ) and 0.779 (sensitivity $71.3 \%$, specificity $70.6 \%$ ) in the test and validation cohorts, respectively. In addition, ROC curve analysis revealed the sensitivity of miR-106b levels as a diagnostic indicator of ESCC (Fig. 2C and F). The AUC was 0.716 (sensitivity 65.5\%, specificity $61.6 \%$ ) and 0.815 (sensitivity $74.3 \%$, specificity $73.5 \%$ ) in the test and validation cohorts, respectively.

Diagnostic ability of miR-1246/miR-106b ratio. We examined the diagnostic ability of the ratio of miR-1246 whose expression was elevated and miR-106b whose expression was reduced in patient sera. We found that the ratio of miR-1246/miR-106b had indeed, a better AUC of 0.901 (sensitivity $80.0 \%$, specificity $80.0 \%$ ) and 0.903 (sensitivity $82.1 \%$, specificity $82.3 \%$ ) in the test and validation cohorts, respectively (Fig. 3A-F).

Relationship between miR-1246 and miR-106b levels in ESCC. A correlation between the expression levels of miR-1246 and miR-106b was evaluated for patients with ESCC in the validation cohort. However, no significant correlation was observed between the respective expression levels (Fig. 4).

Relationship between the miR-1246/106b ratio and clinicopathological factors of ESCC. Statistical analysis was performed to determine the relationships between miR-1246/miR-106b levels and clinicopathological factors of ESCC (Table II). Patient samples were based on the median miR-1246/106b expression levels to obtain high and low groups. High serum miR-1246/106b expression showed a significant association with tumor depth, positive lymph node metastasis, stage, and survival of patients.

Association between miR-1246 and miR-106b expression levels or miR-1246/106b ratio and ESCC prognosis. Overall survival analysis was performed using the Kaplan-Meier approach, with statistical analysis performed using the log-rank test. Using the patient groups described above, the prognostic values of miR-1246, miR-106b, and miR-1246/miR-106b expression levels were evaluated (Fig. 5). The prognosis of the group with high serum miR-1246 expression was significantly worse than that of the group with low serum miR-1246 expression $(\mathrm{P}<0.001)$. The 5-year overall survival rates were 76.1 and $47.6 \%$ for the groups with high and low miR-1246 expression, respectively. The 5-year disease free survival rates were 86.6 and $49.4 \%$ for the groups with high and low miR-1246 expression, respectively. In contrast, there was no association between the serum levels of miR-106b expression and prognosis. In addition, the miR-1246/miR-106b ratio showed an association with prognosis as well as miR-1246. The 5-year overall survival rates were 74.5 and $45.6 \%$ for the groups with high and low miR-1246/miR-106b ratio, respectively. Furthermore, the 5-year disease free survival rates were 85.7 and $51.0 \%$ for the groups with high and low $\mathrm{m}$ iR-1246/miR-106b ratio, respectively.

\section{Discussion}

There have been numerous reports on the expression of miRs being associated with cancer and its significance $(23,24)$. Many studies have also been reported on the expression of miRs in body fluids including serum (25). The mechanism by which miRs are stably present in the serum is thought to be related to protection from RNase degradation by being sequestered in exosomes or forming a protein complex with argonaute 2 protein (26-28). In this study, we performed a comprehensive analysis of serum levels of miRs in patients with ESCC that differ from healthy subjects using microarrays. We observed that the expression of multiple miRs was significantly increased or attenuated. Similar observations have been previously reported using serum from patients with other cancer types $(29,30)$. Here, we focused on miR-1246, a $\mathrm{miR}$ with increased expression, and miR-106b, a miR with attenuated expression.

miR-1246 was shown to have elevated serum levels in patients with ESCC in our previous study. Since then, other researchers have reported increased serum levels of this miR in other carcinomas $(31,32)$. In a systematic review of gastrointestinal cancer, it was reported that the expression of 

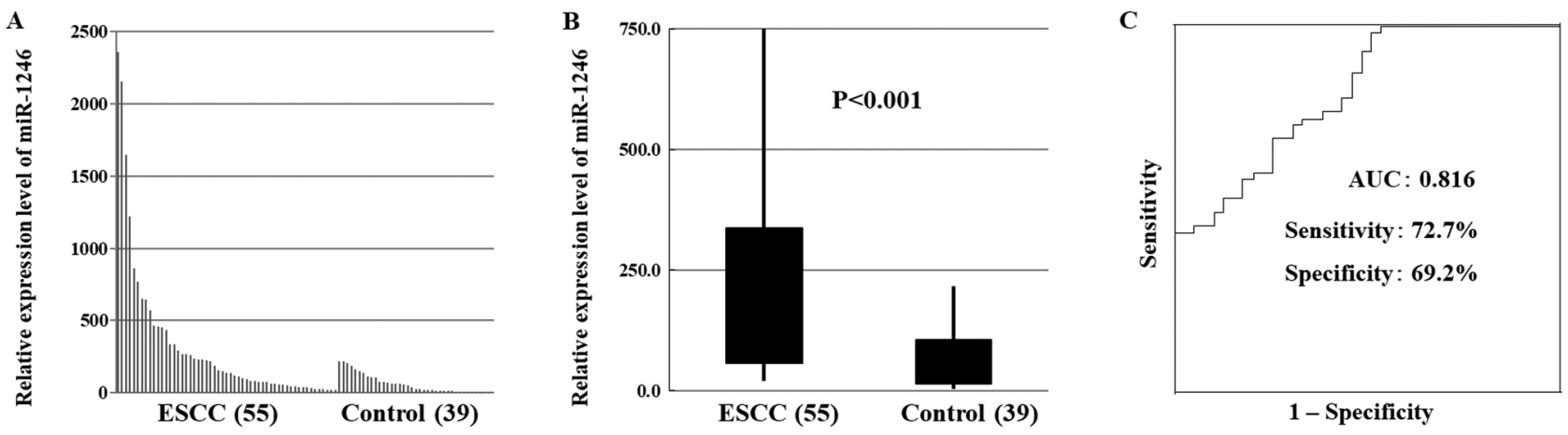

1 - Specificity
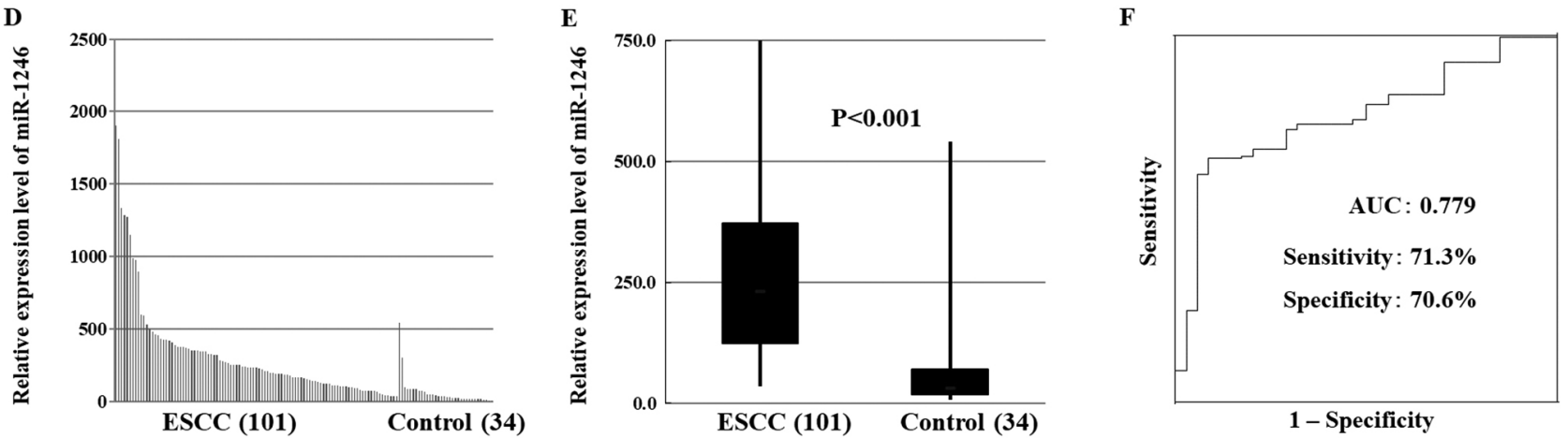

Figure 1. Serum miR-1246 expression in patients with ESCC. (A) In the test cohort, miR-1246 expression was increased in the serum of patients with ESCC compared with healthy controls. (B) In the test cohort, miR-1246 expression was statistically significantly higher in patients with ESCC compared with healthy controls $(\mathrm{P}<0.001)$. (C) Receiver operating characteristic curve analysis revealed the sensitivity of miR-1246 as a diagnostic indicator of ESCC in the test cohort. (D) In the validation cohort, miR-1246 expression was increased in the serum of patients with compared with healthy controls. (E) In the validation cohort, miR-1246 expression was statistically significantly higher in patients with ESCC compared with healthy controls $(\mathrm{P}<0.001)$. (F) Receiver operating characteristic curve analysis revealed the sensitivity of miR-1246 as a diagnostic indicator of ESCC in the validation cohort. miR, microRNA; ESCC, esophageal squamous cell carcinoma; AUC, area under the curve.
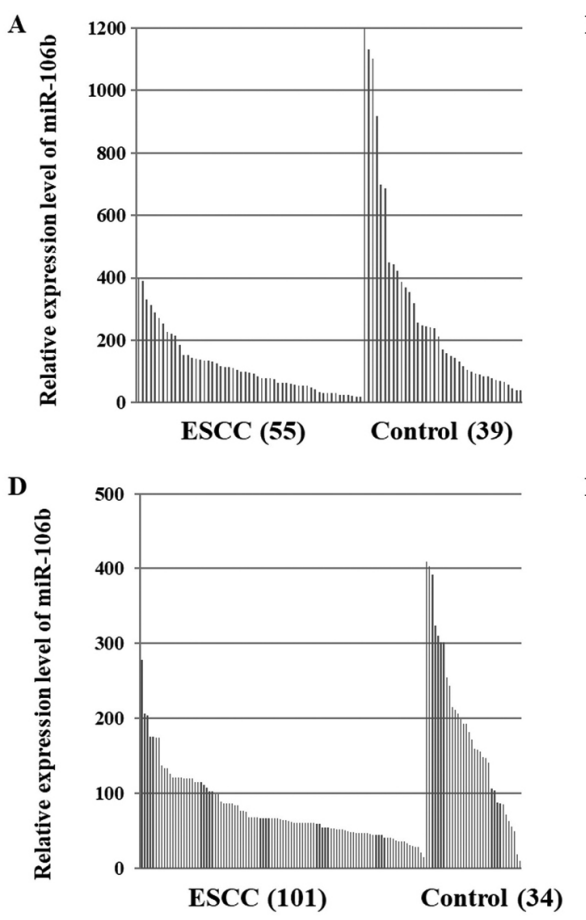

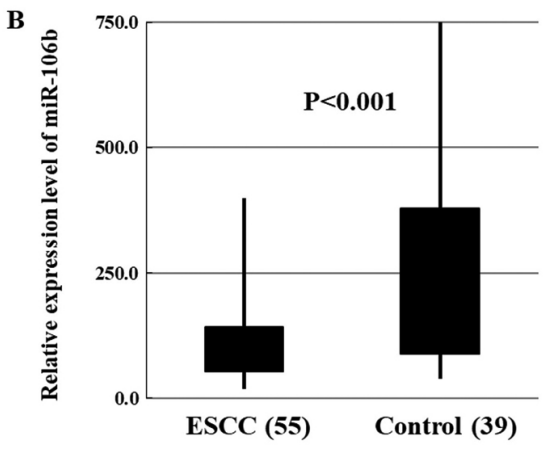

$\mathbf{E}$

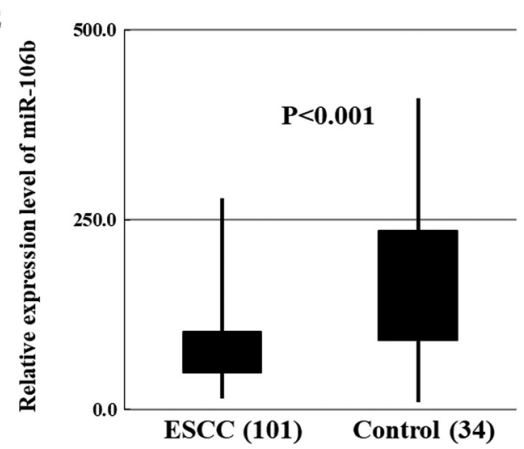

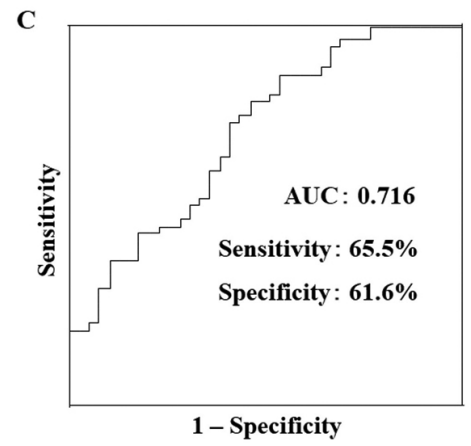

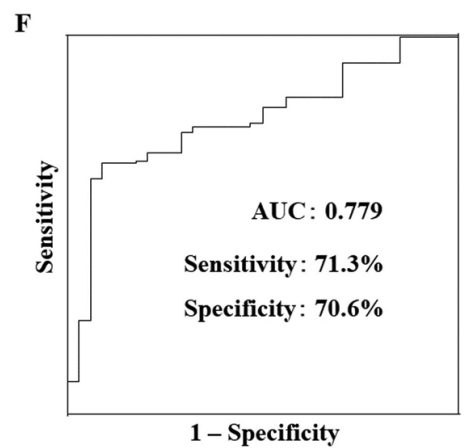

Figure 2. Serum miR-106b expression in patients with ESCC. (A) In the test cohort, miR-106b expression was decreased in the serum of patients with ESCC compared with healthy controls. (B) In the test cohort, miR-106b expression was statistically significantly decreased in patients with ESCC compared with healthy controls $(\mathrm{P}<0.001)$. (C) In the test cohort, receiver operating characteristic curve analysis revealed the sensitivity of miR-106b as a diagnostic indicator of ESCC. (D) In the validation cohort, miR-106b expression was decreased in the serum of patients with ESCC compared with healthy controls. (E) In the validation cohort, miR-106b expression was statistically significantly decreased ESCC patients than in healthy controls. (F) In the validation cohort, receiver operating characteristic curve analysis revealed the sensitivity of miR-106b as a diagnostic indicator of ESCC. miR, microRNA; ESCC, esophageal squamous cell carcinoma; AUC, area under the curve; miR, microRNA; ESCC, esophageal squamous cell carcinoma; AUC, area under the curve. 

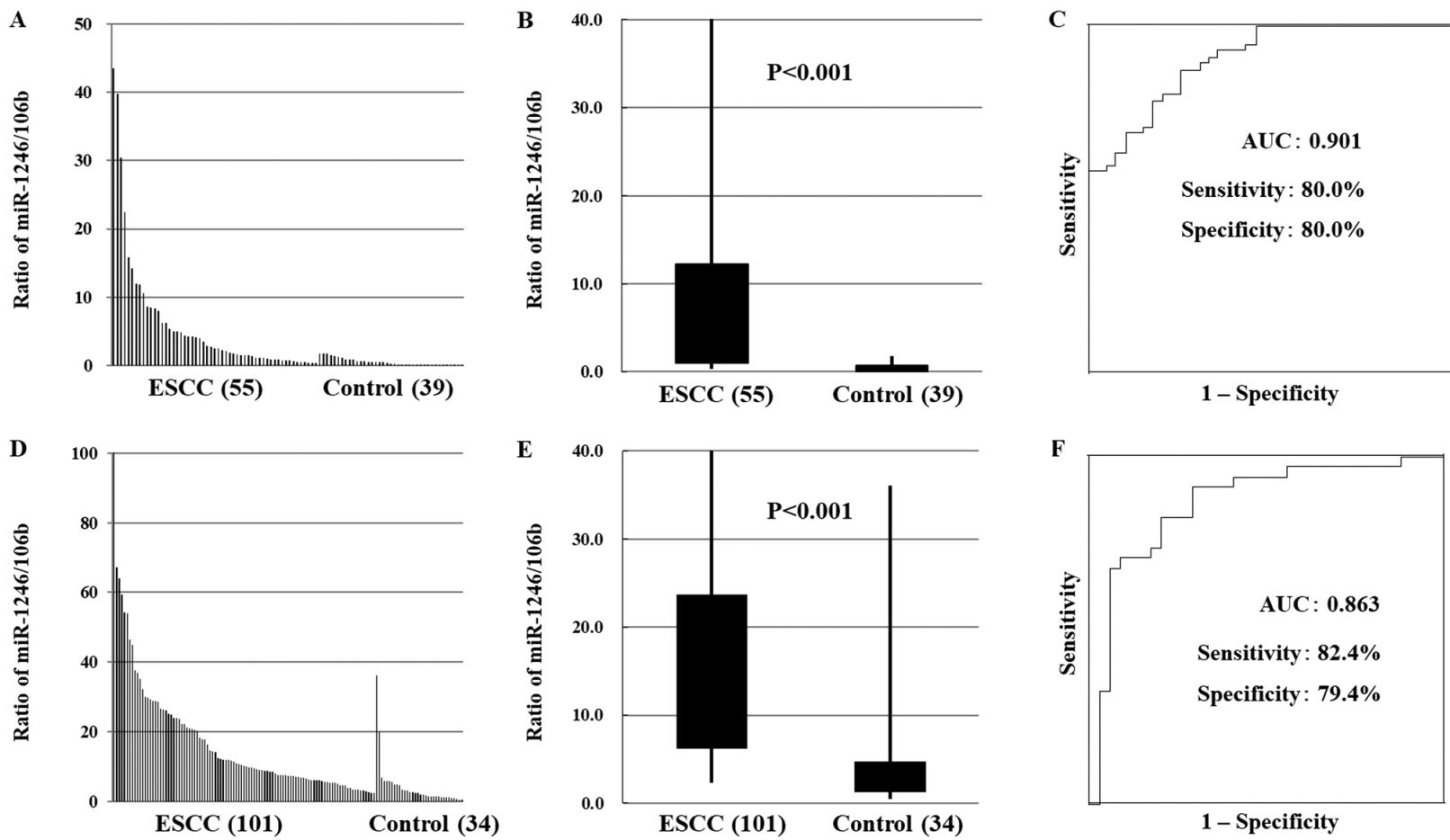

Figure 3. Serum miR-1246/106b ratio in patients with ESCC. (A) In the test cohort, miR-1246/106b ratio was higher in the serum of ESCC patients than in that of healthy controls. (B) In the test cohort, miR-1246/106b ratio was statistically significantly higher in ESCC patients than in healthy controls (P<0.001). (C) The AUC value of the miR-1246/miR-106b ratio was as high as 0.901 (sensitivity, 80.0\%; specificity, 80.0\%) in the test cohort. (D) In the validation cohort, miR-1246/106b ratio was higher in the serum of ESCC patients than in that of healthy controls. (E) In the validation cohort, miR-1246/106b ratio was statistically significantly higher in ESCC patients than in healthy controls $(\mathrm{P}<0.001)$. (F) The AUC value of the miR-1246/miR-106b ratio was as high as 0.903 (sensitivity $82.1 \%$, specificity $82.3 \%$ ) in the validation cohort and 0.903 (sensitivity, $82.1 \%$; specificity, $82.3 \%$ ) in the validation cohort. miR, micro RNA; ESCC, esophageal squamous cell carcinoma; AUC, area under the curve.

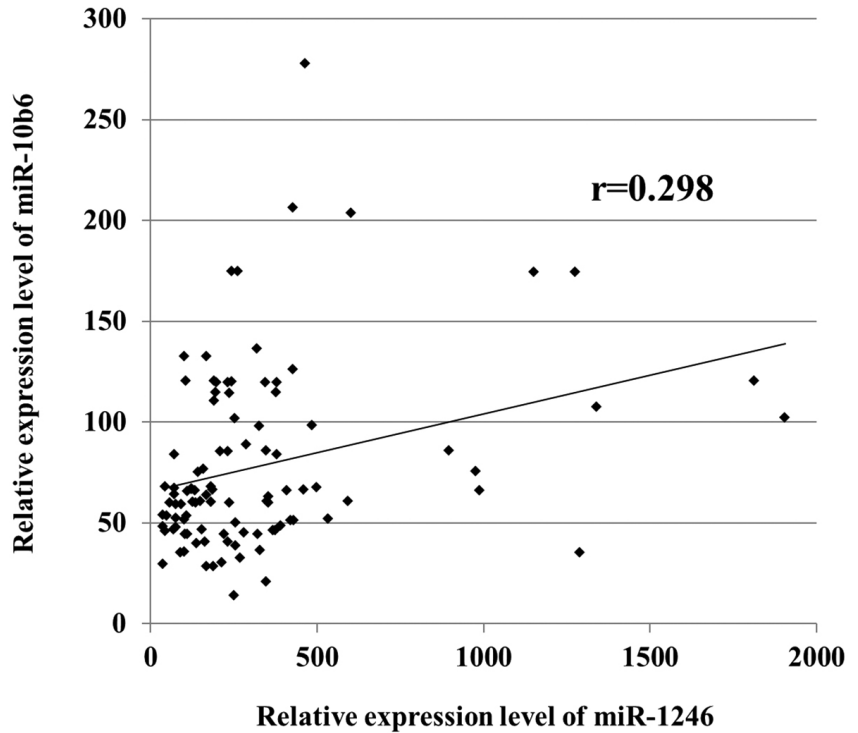

Figure 4. Correlation between miR-1246 and miR-106b expression. No significant correlation was observed between the respective levels of miR-1246 and miR-106b. miR, microRNA.

miR-1246 in serum is more useful as a biomarker than that of miR-21, which is an oncomiR (12). Moshiri et al demonstrated the utility of plasma and serum miR biomarkers in hepatocellular carcinoma using combinations of multiple miRs including miR-1246. However, their analysis included a single cohort, and it is considered that validation using other cohorts is required (33). Therefore, here we used different cohorts to increase the reliability of the findings. The function of miR-1246 in cancer has been reported so far in studies using cancer tissues and cell lines. In a report using serum from 11 patients with lung cancer, miR-1246 was the circulating miR with the highest expression compared with healthy subjects. In addition, in studies using A549, a lung cancer cell line, miR-1246 regulates the $\mathrm{Wnt} / \beta$-catenin pathway by targeting GSK-3 $\beta / \beta$-catenin, thereby regulating cell migration ability and metastatic potential (34). In addition, miR-1246 is implicated in curcumin's anticancer effect and attenuated radiation sensitivity. It has been stated that miR-1246 directly targets p53 and inhibits p53 translation (35). In uterine cervical cancer, miR-1246 has been reported to inhibit thrombospondin-2, which regulates cell adhesion and migration via hydrolysis of the extracellular matrix and a signaling pathway $(36,37)$. From the above reports, it is presumed that miR-1246 functions as an oncomiR, and its expression may be increased in the serum of ESCC patients.

miR-106b-5p has been identified as an oncogenic miR in hepatocellular carcinoma, laryngeal carcinoma, breast carcinoma, and glioma (38). miR-106b is present in the intron of the MCM7 gene and forms a cluster with miR-25 and miR-93 $(39,40)$. Reports on the function of miR-106b are controversial, wherein few report its tumor suppressive function in colorectal and ovarian cancers while others report its action as an oncomir in colorectal and other cancers. Functionally silencing ectopic or endogenous miR-106b-5p expression inhibited or promoted colorectal cancer (CRC) cell invasion and metastasis in vitro 
Table II. Correlation between the 1246/106b ratio and the clinicopathological features of patients with ESCC.

\begin{tabular}{|c|c|c|c|c|}
\hline Characteristics & Total & High $1246 / 106 b$ ratio & Low $1246 / 106 b$ ratio & $\mathrm{P}$-value \\
\hline Total & 101 & $51(50.5)$ & $50(49.5)$ & \\
\hline Sex & & & & 0.5564 \\
\hline Male & 85 & $44(43.6)$ & $41(40.6)$ & \\
\hline Female & 16 & $7(6.9)$ & $9(8.9)$ & \\
\hline Age, years & & & & 0.1707 \\
\hline$<65$ & 37 & $22(21.8)$ & $15(14.9)$ & \\
\hline$\geq 65$ & 64 & $29(28.7)$ & $35(34.6)$ & \\
\hline Tumor depth & & & & $<0.001$ \\
\hline $\mathrm{T} 1-2$ & 42 & $11(10.9)$ & $31(30.7)$ & \\
\hline $\mathrm{T} 3-4$ & 59 & $40(39.6)$ & $19(18.8)$ & \\
\hline Lymph node metastasis & & & & $<0.001$ \\
\hline Negative & 97 & $18(17.8)$ & $35(34.6)$ & \\
\hline Positive & 4 & $33(32.7)$ & $15(14.9)$ & \\
\hline Stage & & & & $<0.001$ \\
\hline $\mathrm{I}-\mathrm{II}$ & 55 & $19(18.8)$ & $36(35.6)$ & \\
\hline III-IV & 46 & $32(31.7)$ & $14(13.9)$ & \\
\hline Patient survival & & & & $<0.005$ \\
\hline Alive & 59 & $22(21.8)$ & $37(36.6)$ & \\
\hline Dead & 42 & $29(28.7)$ & $13(12.9)$ & \\
\hline
\end{tabular}

Data are provided as n (\%). ESCC, esophageal squamous cell carcinoma.

A
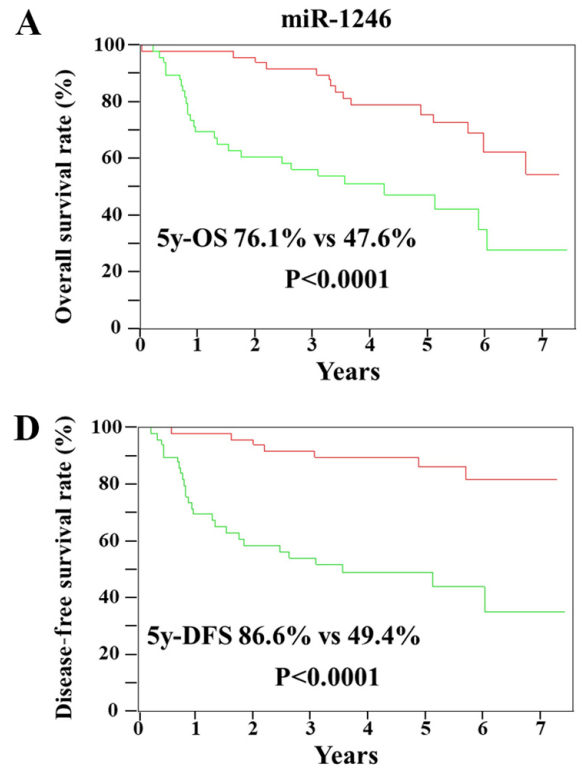

B
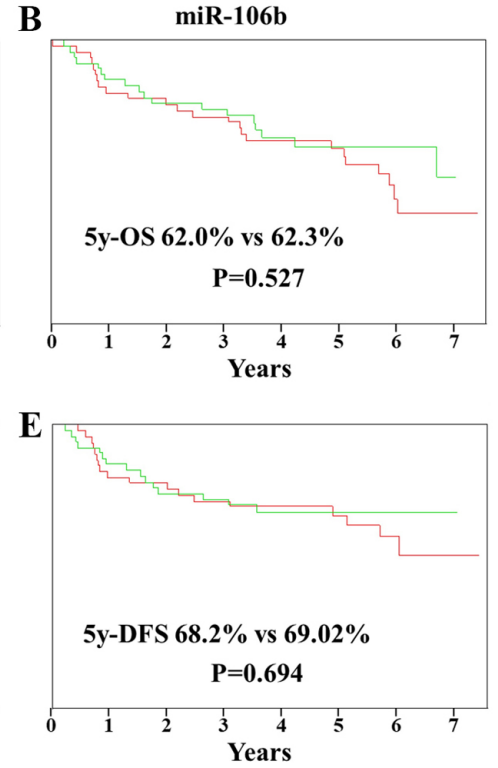

C
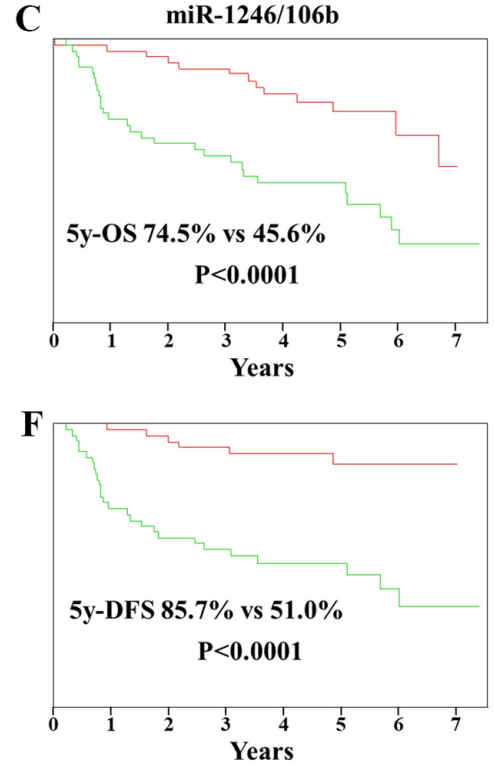

Figure 5. Prognostic analysis of the expression of miR-1246, miR-106b and miR-1246/106b ratio. (A) The 5-year overall survival rates were 76.1 and 47.6\% for the groups with high and low miR-1246 expression, respectively. (B) There was no association between serum miR-106b levels and patient prognosis. (C) The 5-year overall survival rates were 74.5 and $45.6 \%$ for the groups exhibiting high and low miR-1246/miR-106b ratios, respectively. (D) The 5-year disease free survival rates were 86.6 and $49.4 \%$ for the groups with high and low miR-1246 expressions, respectively. (E) There was no association between serum miR-106b expression and patient prognosis. (F) The 5-year disease free survival rates were 85.7 and 51.0\% for the groups with high and low miR-1246/miR-106b ratios, respectively. miR, microRNA; y, year; OS, overall survival; DFS, disease free survival.

and in vivo. The long non-coding RNA MALAT1 mediates SLAIN2-related MT motility and regulates CRC progression by regulating miR-106b-5p expression and functioning as a competing endogenous RNA in vitro and in vivo (21). However, in our study, the expression of miR-93 in the same cluster as miR-106b was attenuated. In this regard, expression of the host of miR-25, miR-93, and miR-106b clusters, and MCM7 is attenuated in patients with ESCC, resulting in attenuated 
expression of these miRs that are located in the MCM7 intron. However, RT-qPCR showed that MCM7 expression increased in ESCC tissues compared with healthy tissues (Fig. S1A). Furthermore, no significant change in tissue expression of any miRs in the miR-25-93 cluster present in the intron of MCM7 was observed (supplementary Fig. S1B-D). There are many reports on increased and decreased miR serum levels, in addition to our report on miRs with changes in expression levels in the serum identified by comprehensive analysis using microarray. However, no paper mentions the cause of the attenuation. We suggest that the cause of attenuated miR-106b expression in ESCC patients may be that miR-106b target genes are increased, thereby activating miR-106b consumption in tissues. The gene expression profile in tissues was compared using microarrays between the high and low miR-106b expression groups. We observed that the expression levels of target genes (programmed cell death 1 ligand 2, hematological and neurological expressed 1, BTG family member 3, etc.) of miR-106b were increased in the low miR-106b expression group (data not shown). In other words, it was suggested that low expression of miR-106b in tumors might have increased expression of miR-106b target genes including oncogenes. Alternatively, irrespective of expression in cancer tissue, in patients in the process of developing ESCC, miR-106b expression is attenuated by some mechanism, and thus miR-106b serum levels may be reduced. Nevertheless, further research is required to uncover the mechanism of the reduced expression of miRs in the serum of cancer patients.

Our previous paper reported that the expression of miR-1246 in the serum of patients with ESCC was an independent prognostic factor (11). However, the AUC on the ROC curve was 0.754 , and both sensitivity and specificity were $\sim 70 \%$. Therefore, we decided to focus on miRs, which decreases in serum of patients with ESCC. However, as expected, miR-106b had a significant decrease in its expression in the serum, but the AUC was $\sim 0.7$ and it was inferior to that of miR-1246. Therefore, we expected that improvement could be obtained by combining miR (miR-1246) that significantly increases and miR (miR-106b) that significantly decreases. Unfortunately, no significant association was found between each expression. However, as a result, it was clarified that the ratio of $\mathrm{miR}-1246 / \mathrm{miR}-106 \mathrm{~b}$ was the most useful with an AUC of $~ 0.9$. On the other hands, regarding the correlation with pathological factors, we showed that the ratio of $\mathrm{miR}-1246 / \mathrm{miR}-106 \mathrm{~b}$ was associated with tumor invasion depth, lymph node metastasis, progression, life and death. Actually, similar results were already obtained in a single report of miR-1246 (11). In other words, it may be sufficient to investigate miR-1246 alone for prognosis and correlation with histopathological factors. However, again, when focusing on the usefulness as a biomarker, the sensitivity and specificity of miR-1246 alone was $\sim 70 \%$, but by using the ratio of miR-1246 and miR-106b was $\sim 80 \%$. In other words, we believe that the $\mathrm{miR} 1-1246 / \mathrm{miR}-106 \mathrm{~b}$ ratio is useful for distinguishing between ESCC patients and healthy subjects.

However, although this combination improved the diagnostic ability in the present study, a clear improvement in the accuracy as a prognostic marker was not confirmed.

Our study has several limitations. We used sera obtained at multiple facilities, but it is a retrospective study, and we considered it necessary to verify the findings of this study by a prospective study that includes other facilities. The reliability of $\mathrm{miR}$ whose expression is reduced in serum was clarified using multiple samples, but the underlying mechanism remains unclear, which should be addressed in future studies. Furthermore, in ESCC, early detection is directly associated with a good prognosis, but the present findings did not show its usefulness in early diagnosis.

In this study, we reported the usefulness of serum levels of miR-1246 and miR-106b in ESCC patients. In addition, it has been clarified that the diagnostic ability can further be improved by using the ratio of a miR having higher expression to a miR having lower expression. We suggest that such a combination of miRs in the serum may be used clinically as a screening marker in the future.

\section{Acknowledgements}

Not applicable.

\section{Funding}

This work was supported by Japan Society for the Promotion of Science KAKENHI for scientific research (grant no. 17K10616) and Cancer Research Funds for Patients and Family (grant no. H30-11).

\section{Availability of data and materials}

The datasets used and/or analyzed during the current study are available from the corresponding author on reasonable request.

\section{Authors' contributions}

IH planned, analyzed and conducted all experiments and wrote the manuscript. FI, YI, HG and NK performed RT-qPCR and analyzed resulting data. FS, YN NT and HM contributed to study conception and design, acquisition of data and the analysis and interpretation of data. FI and YI acquired, analyzed and interpreted the data, drafted the manuscript and revised it critically for important intellectual content. All authors read and approved the final manuscript.

\section{Ethics approval and consent to participate}

The present study was approved by Ethical Committees of the Graduate School of Medicine, Chiba University (approval no. 889), and the Chiba Cancer Center (grant no. H29-0005). All procedures were performed in accordance with the ethical standards of the responsible committee on human experimentation and with the Helsinki Declaration of 1964 and its later amendments. Informed consent was obtained from all patients.

\section{Patient consent for publication}

Written informed consent was obtained from the patients for the publication of the current study and accompanying clinicopathological data. 


\section{Authors' information}

Not applicable.

\section{Competing interests}

The authors declare that they have no competing interests.

\section{References}

1. Bray F, Ferlay J, Soerjomataram I, Siegel RL, Torre LA and Jemal A: Global cancer statistics 2018: GLOBOCAN estimates of incidence and mortality worldwide for 36 cancers in 185 countries. CA Cancer J Clin 68: 394-424, 2018.

2. Brown LM, Devesa SS and Chow WH: Incidence of adenocarcinoma of the esophagus among white Americans by sex, stage, and age. J Natl Cancer Inst 100: 1184-1187, 2008.

3. Kamangar F, Dores GM and Anderson WF: Patterns of cancer incidence, mortality, and prevalence across five continents: Defining priorities to reduce cancer disparities in different geographic regions of the world. J Clin Oncol 24: 2137-2150, 2006.

4. Law S and Wong J: The current management of esophageal cancer. Adv Surg 41: 93-119, 2007.

5. Headrick JR, Nichols FC III, Miller DL, Allen MS, Trastek VF, Deschamps C, Schleck CD, Thompson AM and Pairolero PC: High-grade esophageal dysplasia: Long-term survival and quality of life after esophagectomy. Ann Thorac Surg 73: 1697-1703, 2002

6. Lin DC, Hao JJ, Nagata Y, Xu L, Shang L, Meng X, Sato Y, Okuno Y, Varela AM, Ding LW, et al: Genomic and molecular characterization of esophageal squamous cell carcinoma. Nat Genet 46: 467-473, 2014.

7. Cancer Genome Atlas Research Network; Analysis Working Group: Asan University; BC Cancer Agency; Brigham and Women's Hospital; Broad Institute; Brown University; Case Western Reserve University; Dana-Farber Cancer Institute; Duke University; Greater Poland Cancer Centre, et al: Integrated genomic characterization of oesophageal carcinoma. Nature 541: 169-175, 2017.

8. Lee RC, Feinbaum RL and Ambros V: The C. Elegans heterochronic gene lin-4 encodes small RNAs with antisense complementarity to lin-14. Cell 75: 843-854, 1993.

9. Ruvkun G: Molecular biology. Glimpses of a tiny RNA world. Science 294: 797-799, 2001.

10. Calin GA and Croce CM: MicroRNA signatures in human cancers. Nat Rev Cancer 6: 857-866, 2006.

11. Takeshita N, Hoshino I, Mori M, Akutsu Y, Hanari N, Yoneyama Y,Ikeda N, Isozaki Y, Maruyama T, Akanuma N, et al: Serum microRNA expression profile: miR-1246 as a novel diagnostic and prognostic biomarker for oesophageal squamous cell carcinoma. Br J Cancer 108: 644-652, 2013.

12. Wei C, Li Y, Huang K, Li G and He M: Exosomal miR-1246 in body fluids is a potential biomarker for gastrointestinal cancer. Biomark Med 12: 1185-1196, 2018.

13. Ni S, Weng W, Xu M, Wang Q, Tan C, Sun H, Wang L, Huang D, Du $X$ and Sheng W: miR-106b-5p inhibits the invasion and metastasis of colorectal cancer by targeting CTSA. OncoTargets Ther 11: 3835-3845, 2018.

14. Li N, Miao Y, Shan Y, Liu B, Li Y, Zhao L and Jia L: miR-106b and miR-93 regulate cell progression by suppression of PTEN via PI3K/Akt pathway in breast cancer. Cell Death Dis 8: e2796, 2017.

15. Chen S, Chen X, Xiu YL, Sun KX and Zhao Y: Inhibition of ovarian epithelial carcinoma tumorigenesis and progression by microRNA $106 \mathrm{~b}$ mediated through the RhoC pathway. PLoS One 10: e0125714, 2015.

16. Shen G, Jia H, Tai Q, Li Y and Chen D: miR-106b downregulates adenomatous polyposis coli and promotes cell proliferation in human hepatocellular carcinoma. Carcinogenesis 34: 211-219, 2013.

17. Zhang A, Hao J, Wang K, Huang Q, Yu K, Kang C, Wang G, Jia Z, Han L and Pu P: Down-regulation of miR-106b suppresses the growth of human glioma cells. J Neurooncol 112: 179-189, 2013.

18. Xu Y, Wang K, Gao W, Zhang C, Huang F, Wen S and Wang B: MicroRNA-106b regulates the tumor suppressor RUNX3 in laryngeal carcinoma cells. FEBS Lett 587: 3166-3174, 2013.

19. Liu F, Gong J, Huang W, Wang Z, Wang M, Yang J, Wu C, Wu Z and Han B: MicroRNA-106b-5p boosts glioma tumorigensis by targeting multiple tumor suppressor genes. Oncogene 33: 4813-4822, 2014.
20. Prasad R and Katiyar SK: Down-regulation of miRNA-106b inhibits growth of melanoma cells by promoting G1-phase cell cycle arrest and reactivation of $\mathrm{p} 21 / \mathrm{WAF} 1 / \mathrm{Cip} 1$ protein. Oncotarget 5: 10636-10649, 2014.

21. Zhuang M, Zhao S, Jiang Z, Wang S, Sun P, Quan J, Yan D and Wang X: MALAT1 sponges miR-106b-5p to promote the invasion and metastasis of colorectal cancer via SLAIN2 enhanced microtubules mobility. EBioMedicine 41: 286-298, 2019.

22. Livak KJ and Schmittgen TD: Analysis of relative gene expression data using real-time quantitative PCR and the 2(-Delta Delta C(T)) method. Methods 25: 402-408, 2001.

23. Hoshino I and Matsubara H: MicroRNAs in cancer diagnosis and therapy: From bench to bedside. Surg Today 43: 467-478, 2013.

24. Hermeking H: MicroRNAs in the p53 network: Micromanagement of tumour suppression. Nat Rev Cancer 12: 613-626, 2012.

25. Schwarzenbach H, Nishida N, Calin GA and Pantel K: Clinical relevance of circulating cell-free microRNAs in cancer. Nat Rev Clin Oncol 11: 145-156, 2014.

26. Skog J, Würdinger T, van Rijn S, Meijer DH, Gainche L, Sena-Esteves M, Curry WT Jr, Carter BS, Krichevsky AM and Breakefield XO: Glioblastoma microvesicles transport RNA and proteins that promote tumour growth and provide diagnostic biomarkers. Nat Cell Biol 10: 1470-1476, 2008.

27. Hayes J, Peruzzi PP and Lawler S: MicroRNAs in cancer: Biomarkers, functions and therapy. Trends Mol Med 20: 460-469, 2014.

28. Khoury S and Tran N: Circulating microRNAs: Potential biomarkers for common malignancies. Biomark Med 9: 131-151, 2015.

29. Kumar S, Sharawat SK, Ali A, Gaur V, Malik PS, Kumar S, Mohan A and Guleria R: Identification of differentially expressed circulating serum microRNA for the diagnosis and prognosis of Indian non-small cell lung cancer patients. Curr Probl Cancer 44: 100540, 2020.

30. Yoshida K, Yokoi A, Kagawa T, Oda S, Hattori S, Tamauchi S, Ikeda Y, Yoshikawa N, Nishino K, Utsumi F, et al: Unique miRNA profiling of squamous cell carcinoma arising from ovarian mature teratoma: Comprehensive miRNA sequence analysis of its molecular background. Carcinogenesis 40: 1435-1444, 2019.

31. Chuma M, Toyoda H, Matsuzaki J, Saito Y, Kumada T, Tada T, Kaneoka Y, Maeda A, Yokoo H, Ogawa K, et al: Circulating microRNA-1246 as a possible biomarker for early tumor recurrence of hepatocellular carcinoma. Hepatol Res 49: 810-822, 2019.

32. Shi Y, Wang Z, Zhu X, Chen L, Ma Y, Wang J, Yang X and Liu Z: Exosomal miR-1246 in serum as a potential biomarker for early diagnosis of gastric cancer. Int J Clin Oncol 25: 89-99, 2019.

33. Moshiri F, Salvi A, Gramantieri L, Sangiovanni A, Guerriero P, De Petro G, Bassi C, Lupini L, Sattari A, Cheung D, et al: Circulating miR-106b-3p, miR-101-3p and miR-1246 as diagnostic biomarkers of hepatocellular carcinoma. Oncotarget 9: 15350-15364, 2018.

34. Yang F, Xiong H, Duan L, Li Q, Li X and Zhou Y: miR-1246 promotes metastasis and Invasion of A549 cells by targeting GSK-3 $\beta$-mediated Wnt $/ \beta$-catenin pathway. Cancer Res Treat 51: 1420-1429, 2019.

35. Xu R, Li H, Wu S, Qu J, Yuan H, Zhou Y and Lu Q: MicroRNA-1246 regulates the radio-sensitizing effect of curcumin in bladder cancer cells via activating P53. Int Urol Nephrol 51: 1771-1779, 2019.

36. Chen J, Yao D, Zhao S, He C, Ding N, Li L and Long F: miR-1246 promotes $\mathrm{SiHa}$ cervical cancer cell proliferation, invasion, and migration through suppression of its target gene thrombospondin 2. Arch Gynecol Obstet 290: 725-732, 2014.

37. Du P, Lai YH, Yao DS, Chen JY and Ding N: Downregulation of microRNA-1246 inhibits tumor growth and promotes apoptosis of cervical cancer cells by targeting thrombospondin-2. Oncol Lett 18: 2491-2499, 2019.

38. Cai K, Wang Y and Bao X: miR-106b promotes cell proliferation via targeting RB in laryngeal carcinoma. J Exp Clin Cancer Res 30: 73, 2011.

39. Mehlich D, Garbicz F and Włodarski PK: The emerging roles of the polycistronic miR-106b-25 cluster in cancer - A comprehensive review. Biomed Pharmacother 107: 1183-1195, 2018.

40. Tamilzhalagan S, Rathinam D and Ganesan K: Amplified 7q21-22 gene MCM7 and its intronic miR-25 suppress COL1A2 associated genes to sustain intestinal gastric cancer features. Mol Carcinog 56: 1590-1602, 2017.

This work is licensed under a Creative Commons Attribution-NonCommercial-NoDerivatives 4.0 International (CC BY-NC-ND 4.0) License. 